\title{
An investigation of the partial reinforcement extinction effect in humans and corresponding changes in physiological variables
}

\author{
DAVID J. PITTENGER and WILLIAM B. PAVLIK \\ University of Georgia, Athens, Georgia
}

\begin{abstract}
Human subject's heart rate and skin conductance were monitored during periods of acquisition and extinction. During acquisition, subjects received either $100 \%$ or $50 \%$ reinforcement for correct responses. The behavioral data indicated that subjects exposed to partial reinforcement were more persistent than subjects exposed to continuous reinforcement. Significant increases in skin conductance were observed at the start of acquisition and extinction, but there were no differences between the two reinforcement groups. These data are reviewed with respect to Amsel's frustration-mediation hypothesis of persistence.
\end{abstract}

Amsel's frustration-mediation theory of persistence $(1962,1967,1972)$ states that behavior becomes more resistant to extinction when it is associated with partial reinforcement because of frustration responses that are produced by nonreinforcement. In support of this theory, Daly (e.g., 1974) has demonstrated that neutral cues obtain aversive properties when associated with nonreinforcement. The use of anxiolytic compounds to attenuate the frustrative and aversive properties of nonreinforcement has, however, produced an inconsistent and problematic collection of data. Several researchers have concluded that the anxiolytics have no effect upon persistence (e.g., Demarest \& MacKinnon, 1978; Ziff \& Capaldi, 1971), whereas others have concluded that they reduce or abolish the partial reinforcement extinction effect (PREE) (e.g., Fledon \& Gray, 1981; McNaughton, 1984).

The purpose of the present experiment was to examine, in humans, the effects of continuous (CRF) and partial (PRF) reinforcement schedules on physiological and behavioral variables during periods of training and extinction. Specifically, heart rate (HR) and skin conductance (SC) were monitored as subjects performed a simple, four-alternative, discrete-trial task during periods of acquisition and extinction. Previous research (e.g., Pittenger \& Pavlik, 1988) had demonstrated that a large and reliable PREE is produced using this task.

Germana and Pavlik (1964) measured skin conductance responses (SCRs) while human subjects performed a simple instrumental task during periods of acquisition and extinction. During acquisition, the SCR amplitude was greatest on the trial when the subjects made the first correct response, and the amplitude of subsequent SCRs diminished over additional trials. In the first extinction trial, it was found that the amplitude of the SCR was a

Requests for reprints may be addressed to the first author, Department of Psychology, Marietta College, Marietta, OH 45750. direct and positive function of the number of prior reinforcements received by the subject. On the basis of these data and Amsel's frustration theory, we made several predictions. During the acquisition period, physiological responses (e.g., changes in SCR and HR) should be greater for subjects exposed to PRF than for subjects exposed to CRF. In contrast, at the start of extinction, the responses should be greater for subjects exposed to CRF than for subjects exposed to PRF, because the anticipatory goal response, and thus frustration, will be greatest for the CRF group. As extinction progresses, the magnitude of the physiological responses of subjects exposed to PRF should increase as the frustrative effects of nonreinforcement reemerge.

\section{METHOD}

\section{Subjects}

The subjects were 32 (16 male and 16 female) students from the University of Georgia who volunteered to participate in the experiment in exchange for course credit.

\footnotetext{
Apparatus

Behavioral measure. The Human Shuttle Box (HSB) task, described previously (Pittenger \& Pavlik, 1988), was used. This is a simple video game that, for reinforcement, requires the subject to make one of four possible responses on each trial. In the present experiment, continuous reinforcement was based on a $100 \%$ probability that the word "correct" would appear contingent upon the subject's making the criterion response. Partial reinforcement was defined as a $50 \%$ probability of reinforcement for contingent responses. In addition, all subjects were told that there was a $\$ 50$ prize for the subject making the greatest number of correct responses.

Physiological measures. To measure heart rate, electrodes were placed in a Lead III (left arm-left leg) configuration on the subject. The signal obtained was filtered and amplified by a Grass 7P4 preamplifier and recorded directly on chart paper, along with SC, for later analysis. The heart rate was determined on a beat-by-beat basis by measuring the interbeat interval (IBI).

To measure SC, $\mathrm{Ag} / \mathrm{AgCl}$ electrodes, placed on the medial phalanx of the middle and ring fingers of the subject's left hand, a constant voltage circuit (0.5 V; Lykken \& Venables, 1971; Venables \& Christie, 1973), and a Grass 7P-1 preamplifier were used. For each trial, the SCR
} 


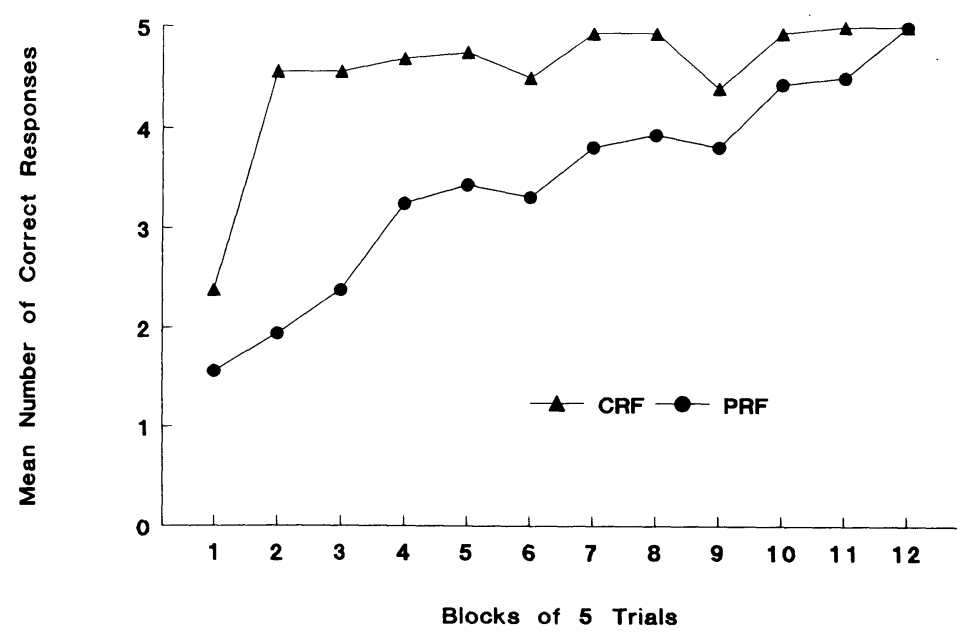

Figure 1. Mean number of correct responses made during acquisition over 12 blocks of five trials.

was determined by measuring the change in conductance that occurred after the response was made. On the basis of pilot research, the response latency window was set at $3.0 \mathrm{sec}$ after the end of the postresponse feedback.

\section{Procedure}

After consenting to participate in the experiment, a subject was taken to the experimental chamber and prepared for the physiological recording. The experimenter gave basic instructions on the HSB task (see Pittenger \& Pavlik, 1988, for detailed instructions) and answered any general questions concerning the task, but did not reveal aspects of the experiment concerning either PRF or extinction. The subject was allowed to practice on a simulation of the experiment until he/she had made 10 consecutively correct responses. The subject was then required to sit quietly for $5 \mathrm{~min}$ while heart rate and skin conductance were monitored for baseline purposes. After the baseline period had elapsed, the acquisition phase was begun.

The acquisition phase consisted of 60 trials of the HSB task. For each trial, the subject was allowed $3.5 \mathrm{sec}$ in which to make a response. Failure to respond within this time caused the trial to end and to be scored as a failure to respond. When a response was made, there was a 1.5-sec delay during which the appropriate reinforcement was presented. The intertrial interval consisted of $5.0 \mathrm{sec}$ plus the difference between the subject's latency to respond and the 3.5 -sec criterion. Thus, the interval between the start of each trial was $10.0 \mathrm{sec}$ for all subjects.

Extinction began without warning and immediately following the last trial of acquisition. The extinction session consisted of 60 nonreinforced trials. All other aspects of the experiment remained constant.

\section{RESULTS}

\section{Behavioral Data}

Acquisition. The acquisition data for both groups are presented in Figure 1. As is evident, subjects exposed to CRF achieved an asymptotic level of performance in fewer trials than did subjects exposed to PRF. By the end of the acquisition session, however, both groups were performing at comparable levels. An ANOVA of these data yielded a significant interaction between schedules of reinforcement and blocks of trials $[F(11,330)=5.93$, $p<.05]$. Further analysis of this interaction indicated that the mean number of correct responses of the CRF group was reliably greater than that of the PRF group only during the first five blocks of trials.

Extinction. The extinction data were transformed using Anderson's (1963) rate transformation, with the average of the last three blocks of acquisition serving as the baseline. These data are presented in Figure 2. The pattern of results is consistent with those found earlier (cf. Pittenger \& Pavlik, 1988): There is a rapid decrease in the overall production of persistent responses over the first 15 trials of extinction, with subjects exposed to CRF evidencing less resistance to extinction than the PRF subjects. Furthermore, during the subsequent extinction trials, the proportion of persistent responses does not continue to decline, but appears to become more variable and to increase.

Analysis of these data yielded a significant interaction between schedules of reinforcement and blocks of trials $[F(12,360)=2.67, p<.05]$. This interaction indicates that the subjects in the PRF group made more persistent responses within all blocks of trials except for the 2nd, 3rd, and 10th blocks.

\section{Physiological Data}

Heart rate. The HR data for the acquisition and extinction sessions were analyzed by an analysis of covariance that compared (1) schedule of reinforcement, (2) gender of subject, and (3) blocks of trials. For both analyses, the covariate was the mean IBI determined from the baseline period $1 \mathrm{~min}$ prior to the first acquisition trial. No systematic changes in heart rate were found to occur during either phase of the experiment $(p>.05)$.

Skin conductance. SCRs were averaged across blocks of five trials for the acquisition and extinction phases, and subjected to a logarithmic transformation to maximize homogeneity of variance. The transformed data are presented in Figure 3. In brief, it is evident from these data that there was an increase in conductance amplitude 


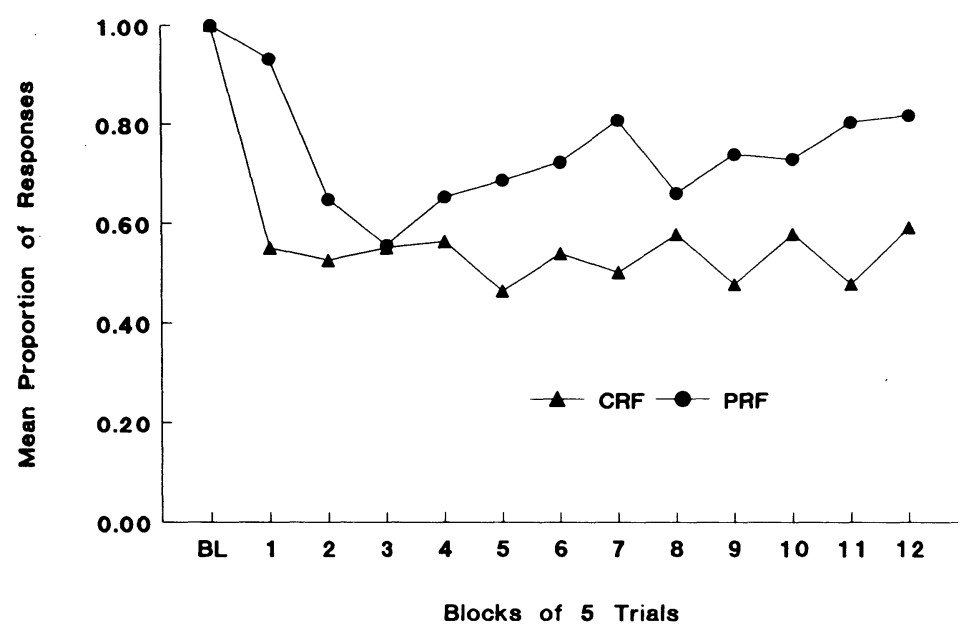

Figure 2. Mean proportion of persistent responses made during a baseline period in acquisition and across 12 blocks of five trials.

during the first block of acquisition for both treatment groups. As acquisition continued, the amplitude of the SCRs decreased until, by the end of the session, the magnitude of the responses was relatively small. An increase in SCR amplitude was also observed to occur during the first several blocks of the extinction session. Over additional extinction trials, the magnitude of the SCRs decreased to minimal levels. These observations were confirmed by an ANOVA of the data.

Separate ANOVAs were conducted on the acquisition and extinction phases of the experiment. For both tests, the factors were schedule of reinforcement and blocks of trials. The ANOVA of the acquisition data yielded a significant main effect for blocks of trials $[F(11,330)=9.77$, $p<.05]$. Subsequent analysis of this effect indicated that by the end of the fourth block of trials there were no significant differences in the mean SCRs across trials. The other components of the ANOVA did not indicate significant effects $(F<1)$.

For the extinction analysis, the last block of acquisition was compared in addition to the extinction data. Analysis of these data yielded a significant main effects for blocks of trials $[F(12,320)=4.911, p<.05]$. This result confirms that there was a significant increase in SCRs across the first three blocks of extinction. By the fourth block of extinction, no further significant changes in SCRs were observed. Neither the schedules factor nor the interaction term produced significant effects.

\section{DISCUSSION}

An attempt was made in this experiment to determine if changes observed in the physiological variables were commensurate in acquisition and extinction phases, and if these changes would follow a pattern suggested by Amsel's $(1962,1967,1972)$ theory of persistence. From the

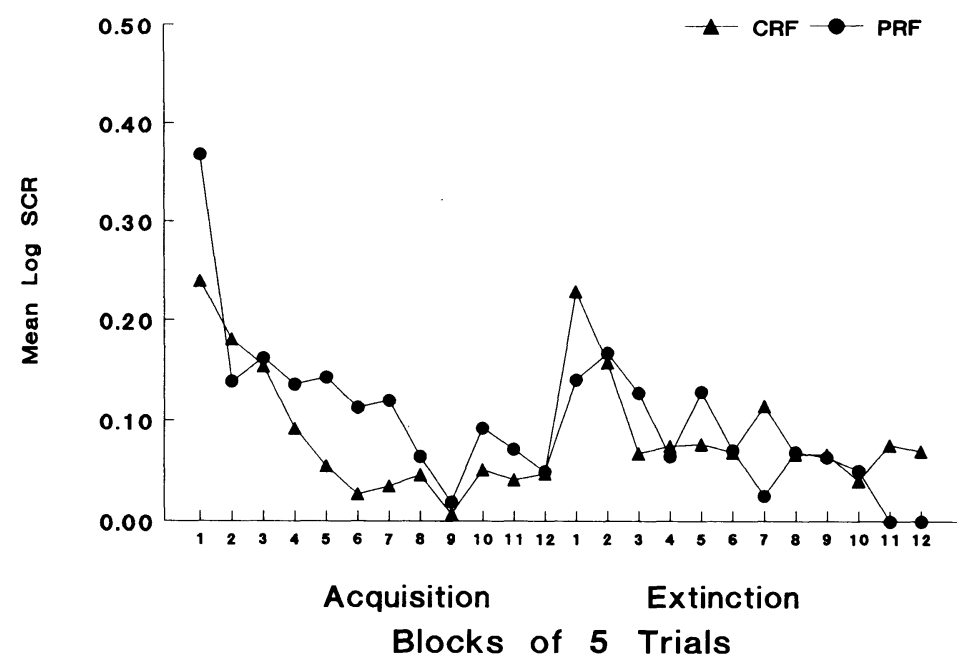

Figure 3. Mean log skin conductance response recorded during periods of acquisition and extinction across blocks of five trials. 
behavioral data, it was observed that subjects exposed to PRF were more persistent than subjects exposed to CRF. There were, however, no systematic differences in the physiological measures between these groups during either acquisition or extinction.

An alternative hypothesis for the lack of observed changes in physiological factors is that the experimental treatment did not create effects sufficient to produce observable changes. In defense of the method used, several general points need to be made. First, the treatments did produce a reliable PREE in the behavior of the subjects. Second, reliable changes in SCRs, albeit small, were observed during the initial trials of acquisition and extinction. Indeed, these changes in SCRs appear to follow the general trends of the two sessions. That is, the time during which the largest changes in SC occurred was the same as that in which the largest changes in performance occurred. Thus, the experiment was successful in producing changes in both behavioral and physiological responses, but the latter were unrelated to Amsel's $(1962,1967,1972)$ theory.

In keeping with these comments, several tentative conclusions can be drawn from the data. Specifically, the changes in SCR amplitude cannot be attributed to frustration responses associated with nonreinforcement. If the SCRs had been influenced by the anticipatory frustration response, the pattern of responses predicted above would have emerged. In contrast, the present data indicate that, in both acquisition and extinction, the subjects' physiological reactions were equivalent regardless of reinforcement condition. Both groups of subjects, however, evidenced increased SCRs at the start of both phases of the experiment. This finding may suggest that the subjects were equally sensitive to changes in reinforcement conditions (i.e., start of extinction) but, for reasons yet to be identified, produced different behavioral response patterns.

\section{REFERENCES}

AmSEL, A. (1962). Frustrative nonreward in partial reinforcement and discrimination learning: Some recent history and theoretical extension. Psychological Review, 69, 306-328.

AMSEL, A. (1967). Partial reinforcement effect on vigor and persistence: Advances in frustration theory derived from a variety of within-subjects experiments. In K. W. Spence \& J. T. Spence (Eds.), The psychol- ogy of learning and motivation (Vol. 1, pp. 1-65). New York: Academic Press.

AMSEL, A (1972). Behavioral habituation, counterconditioning, and a general theory of persistence. In A. Black \& W. K. Prokasy (Eds.), Classical conditioning (Vol. 2, pp. 409-426). New York: AppletonCentury-Crofts.

ANDERson, N. H. (1963). Comparison of different populations: Resistance to extinction and transfer. Psychological Review, 70, 162-179.

DALY, H. B. (1974). Reinforcing properties of escape from frustration aroused in various learning situations. In G. Bower (Ed.), The psychology of learning and motivation (Vol. 8, pp. 187-231). New York: Academic Press.

Demarest, J., \& MacKinnon, J. R. (1978). Effects of chlordiazepoxide and reward magnitude on the acquisition and extinction of a partially reinforced response. Physiological Psychology, 6, 78-82.

Fledon, J., \& GRAY, J. A. (1981). The partial reinforcement extinction effect after treatment with chlordiazepoxide. Psychopharmacology, 73, 269-275.

Germana, J. J., \& Pavlik, W. B. (1964). Autonomic correlations of acquisition and extinction. Psychonomic Science, 1, 109-110.

LyKKen, D. T., \& Venables, P. H. (1971). Direct measurement of skin conductance: A proposal for standardization. Psychophysiology, 8, 656-672.

McNaughton, N. (1984). Effects of anxiolytic drugs on the partial reinforcement extinction effect in runway and Skinner box. Quarterly Journal of Experimental Psychology, 36B, 319-330.

PitTenger, D. J., \& PAVuK, W. B. (1988). Analysis of the partial reinforcement extinction effect in humans using absolute and relative comparisons of schedules. American Journal of Psychology, 101, 1-14.

Venables, P. H., \& Christie, M. J. (1973). Mechanisms, instrumentation, recording techniques and quantification of responses. In W. F. Prokasy \& D. C. Raskin (Eds.), Electrodermal activity in psychological research. New York: Academic Press.

ZIFF, D. R., \& CAPALDI, E. J. (1971). Amytal and the small trial partial reinforcement effect. Journal of Experimental Psychology, 87, 263-269.

(Manuscript received October 6, 1988.) 